\section{Nueva prueba no invasora para el cáncer de mama}

Los cambios proliferativos en el epitelio de los conductos mamarios son parte intrínseca del desarrollo de cáncer de mama. Esos cambios producen zonas de despolarización eléctrica del epitelio dentro del parénquima mamario, las cuales pueden extenderse hasta la superficie de la piel. La información diagnóstica se obtiene mediante una prueba no invasora basada en la medición de electropotenciales de la mama en la epidermis. En un estudio multicéntrico llevado a cabo en ocho ciudades europeas con 661 mujeres a quienes se les practicó biopsia, se investigó si la medición de actividad eléctrica en las mamas mediante sensores de superficie servía para detectar la diferencia entre neoplasias benignas y malignas. Se desarrolló así un índice de despolarización para la prueba.

Los resultados mostraron de forma muy significativa que los cambios eléctricos progresivos correspondían directamente a las características proliferativas de los tejidos sometidos a biopsia. La información discriminatoria se obtuvo tanto en mujeres premenopáusicas como posmenopáusicas, y el índice no se relacionó con la edad. La mediana del índice de las lesiones benignas no proliferativas fue de 0,398; la de las lesiones benignas proliferativas, de 0,531 ; y la de lesiones cancerosas (carcinoma in situ ductal e invasor), de 0,644. La prueba tuvo su mejor rendimiento en mujeres con lesiones palpables, en las que se obtuvo una especificidad de $55 \%$ con $90 \%$ de sensibilidad cuando en los cálculos se tuvieron en cuenta los índices de despolarización junto con la edad de las pacientes. Los autores opinan que la menor discriminación de lesiones no palpables puede estar relacionada con la dificultad de colocar los sensores en el lugar adecuado.

Esta nueva prueba para el cáncer de mama podría reducir el número de biopsias innecesarias en mujeres con neoplasias benignas. Los resultados son objetivos y se obtienen inmediatamente. Además de que es una prueba no invasora, tampoco expone a los pacientes a radiación ionizante, por lo que podría usarse repetidamente sin peligro alguno. Para mejorar la prueba será preciso seguir estudiando la variabilidad biológica de los fenómenos eléctricos y evaluar su idoneidad diagnóstica repitiéndola en poblaciones con prevalencias menores de cáncer. (Cuzick J, Holland R, Barth B, Davies R, Faupel M, Fentiman I, et al. Electropotential mea- surements as a new diagnostic modality for breast cancer. Lancet 1998;352(9125):359-363.)

\section{Vigilancia internacional de la resistencia antimicrobiana}

La emergencia de nuevas cepas de microorganismos patógenos resistentes a los medicamentos es un problema creciente en todas partes del mundo. En mayo de 1998, la Asamblea Mundial de la Salud hizo hincapié en la necesidad de acción urgente al aprobar una resolución que pide la colaboración internacional para vigilar la resistencia antimicrobiana. Actualmente no existe ningún mecanismo oficial para dar la alerta a otros países cuando en alguno se descubren nuevos fenotipos como, por ejemplo, el de Staphylococcus aureus resistente a la vancomicina. A pesar de que el fenómeno de la resistencia es un gran problema mundial, no hay ningún consenso internacional sobre estándares apropiados para su vigilancia. Tampoco se ha determinado cuáles son los patógenos prioritarios, las mejores metodologías de vigilancia, cómo llevar a cabo el intercambio de datos ni cómo analizarlos e interpretarlos. Es crítico elaborar lineamientos claros que aborden esas lagunas y permitan que los países se beneficien de compartir experiencias.

Se necesita contar con información sobre la resistencia a antimicrobianos tanto a nivel local como nacional e internacional. En el ámbito local, esa información permite garantizar los tratamientos clínicos más apropiados y actualizarlos, educar a los que prescriben medicamentos y guiar las políticas sobre el control de infecciones. En el entorno nacional, se requiere esa información para encauzar la política, poner al día las listas de medicamentos esenciales y establecer normas de tratamiento, así como para evaluar los efectos de las estrategias de intervención. La recopilación de datos sobre este problema a nivel internacional es especialmente importante en el sentido de poder compartir la información sobre la emergencia de resistencia en microorganismos patógenos comunes, y de analizar profundamente el impacto de esa resistencia y de las políticas de control. Además, es una manera de estimular el diálogo entre los países y los encargados de tomar decisiones, y de propiciar el desarrollo de programas educativos y de la investigación. 
La vigilancia internacional requiere instalaciones de laboratorio organizadas en forma de red dentro de la cual puedan compartirse los datos sobre patrones de resistencia para su análisis e interpretación. Las acciones de salud pública deben ser guiadas por información así generada. En algunas infecciones, como las del aparato respiratorio, los resultados de pruebas in vitro no siempre se correlacionan con los resultados in vivo. En esos casos, el enlace de la vigilancia de la resistencia con el monitoreo de resultados clínicos ayudaría a evaluar el impacto de la situación en la morbilidad y la mortalidad así como las actividades de vigilancia. Sin embargo, en muchos países en desarrollo y en países con economías en transición, será necesario fortalecer considerablemente las instalaciones de laboratorio y las redes de información para que puedan participar en las actividades descritas. Desde que se comenzó a promover el manejo sindrómico de las infecciones (manejo clínico del paciente que presenta un síndrome sin confirmación de laboratorio, por ejemplo, en infecciones de transmisión sexual) ha habido una tendencia a menospreciar los laboratorios como innecesarios. No obstante, el manejo sindrómico solo puede aplicarse si se mantiene actualizado mediante datos de vigilancia. Un estudio de las listas de medicamentos esenciales de 120 países indicó que 60 de ellas no se habían actualizado en los últimos cinco años.

La OMS está trabajando con sus Estados Miembros para a) lograr un consenso internacional sobre los estándares de vigilancia que se necesitan; b) crear un ambiente de cooperación entre las naciones; c) mejorar los sistemas nacionales de vigilancia por medio de apoyo en materia de adiestramiento, educación y garantía de la calidad, y d) establecer un depósito de información sobre la resistencia de patógenos clave en todo el mundo. (Williams RJ, Ryan MJ. Surveillance of antimicrobial resistancean international perspective. BMJ 1998;317:65.)

\section{La liberación gradual de yodo en el agua de poblaciones aisladas}

El yodo es un nutriente tan escasamente representado en la cadena alimentaria (en las algas marinas y mariscos) que, aparte de poder vivir en una región costera de la cuenca del Pacífico, no hay ninguna estrategia basada en los alimentos que garantice protección contra los trastornos causados por la carencia de yodo. En lo que a la ingestión de yodo se refiere, si se exceptúan las poblaciones pesqueras esquimales, coreanas y japonesas, y los isleños del Pacífico y otros grupos pequeños, el mundo se divide en dos grandes poblaciones. Un grupo está protegido por programas de yodación de la sal apoyados por mandato legislativo y el otro grupo sufre de deficiencia endémica de yodo. El segundo grupo es mucho más grande que el primero.

El enriquecimiento de la sal con yodo es tan efectiva cuando se lleva a cabo de forma apropiada, que hay quienes claman por la yodación universal de la sal para toda la población del mundo, aun en lugares donde la sal es una industria artesanal local y se necesitaría centralizarla para fundamentar la solución. Aun así, la producción de sal no siempre está centralizada y a menudo se desobedecen las normas de fortificación. Se han realizado estudios de alcance mundial que muestran cuán incompleta e inadecuada puede ser la yodación de los abastecimientos de sal, incluso en naciones donde es obligatoria. En los círculos académicos se han ideado y puesto a prueba opciones creativas y alternativas para mejorar la cobertura de la yodación. Se ha añadido yodo al azúcar, a los fertilizantes y al agua, se ha aplicado por vía intramuscular y oral en enlaces covalentes con los lípidos de aceites, y se ha administrado en programas de nutrición escolar. Si el yodo se comportara como su pariente químico el flúor, sería posible yodar el agua tal como se fluorura. Pero este no es el caso y hay que buscar otros sistemas.

Durante 2 años, el agua se evaluó como vehículo para el yodo en cuatro aldeas de una zona árida del Sudán. Cada población tenía entre 263 y 1657 habitantes, que obtenían el agua potable de pozos con bombas manuales o de pozos tradicionales con ayuda de un balde. En esos lugares se ensayó el uso de matrices de silicona que contenían yodo y lo liberaban lentamente mientras flotaban en el agua. Se usó uno o más de estos módulos como fuente de yodo en cada uno de los pozos locales. No se observó ningún cambio de olor, color ni sabor en las aguas yodadas. Las concentraciones de yodo fueron más altas en las muestras recolectadas de noche que en las recogidas por la mañana. Mientras que las concentraciones de yodo antes de la intervención variaron entre 0,04 y $0,14 \mu \mathrm{mol} / \mathrm{L}$, después del uso de los módulos aumentaron a entre 0,31 y $2,21 \mu \mathrm{mol} / \mathrm{L}$. En los pozos tradicionales el aumento fue menor que en los de bomba manual. Al final del primer año, antes de reemplazar las fuentes de yodo, las concentraciones eran de 0,04 a 0,20 $\mu \mathrm{mol} / \mathrm{L}$, pero aumentaron a entre 0,12 y $2,7 \mu \mathrm{mol} / \mathrm{L}$ 6 meses más tarde y volvieron a las concentraciones de base a final del segundo año.

En este estudio se investigó, además de la duración del efecto del yodo y su impacto dietético, la interpretación de indicadores diagnósticos de la nutrición con yodo. Las concentraciones de yodo en la orina aumentaron de tres a cinco veces en las aldeas tratadas, con mayores aumentos en las que usaban pozos con bombas manuales. La prevalencia general de bocio cambió de $69 \%$ al principio de la intervención a $25 \%$ durante los 2 años, si bien en muchos de 
los habitantes que podrían haberse normalizado la dolencia no se detectaba visualmente, ya que la involución de los tejidos ocurre gradualmente. Un resultado interesante fue que los valores de la hormona estimulante de la tiroides (TSH), anormalmente elevados al principio del estudio, se redujeron de 30 a $15 \%$ en los 2 años y las concentraciones de tiroxina en suero $\left(\mathrm{T}_{4}\right)$ aumentaron mientras que disminuyeron las de triyodotironina $\left(\mathrm{T}_{3}\right)$. Aunque es un procedimiento invasor y más caro que los ensayos de orina, la medición de las hormonas refleja mucho más exactamente el impacto biológico de la intervención y no debe soslayarse.

Los resultados del estudio del Sudán indican que la agregación de yodo al agua de las aldeas es un enfoque viable para combatir los trastornos causados por la carencia de yodo. Además, permitieron observar que todavía no tenemos un índice diagnóstico confiable para monitorear cómo responde el estado de una población con respecto al yodo a las intervenciones de salud pública. Ambos aspectos requieren más estudio. (Solomons NW. Slow-release iodine in local water supplies reverses iodine deficiency disorders: Is it worth its salt? Nutr Rev 1998;56(9):280-281.)

\section{Factores de riesgo de enfermedad cardiovascular en la dieta de mujeres blancas y afroamericanas}

En los Estados Unidos de América, las mujeres que pertenecen a minorías étnicas sufren con mayor frecuencia de enfermedades crónicas y tienen una esperanza de vida más corta que las blancas. La salud de la mujer minoritaria se ve afectada por altas tasas de pobreza, falta de instrucción y acceso limitado a la atención de salud. Según estimaciones de la Oficina del Censo, para el año 2050 casi la mitad de la población del país estará constituida por grupos minoritarios. Actualmente, el afroamericano es el más numeroso y en ese grupo suele haber más mujeres obesas y víctimas de cardiopatía coronaria, hipertensión, diabetes mellitus tipo 2 y ciertos tipos de cáncer. Postulando que las prácticas alimentarias de este grupo étnico quizá contribuyan a la aparición de esas enfermedades crónicas, en este estudio Gates y McDonald compararon los conocimientos, las actitudes y la ingesta de energía y nutrientes relacionados con el riesgo de enfermedad cardiovascular de mujeres afroamericanas y blancas. Con ese fin analizaron información de dos encuestas nacionales realizadas por el Departamento de Agricultura entre 1989 y 1991, las cuales contienen datos transversales representativos de los hogares estadounidenses. En una encuesta se recogió información sobre la ingesta de nutrientes y las características demográficas, socioeconómicas y personales. La otra fue una encuesta telefónica de seguimiento que compiló datos sobre la principal planificadora de las comidas en el hogar, su conocimiento de los alimentos y sus actitudes hacia el consumo de los mismos, las formas de comprarlos y prepararlos y la seguridad alimentaria. Se incluyeron en la muestra 2684 mujeres blancas y 449 afromericanas que respondieron en su totalidad a ambas encuestas y proporcionaron información sobre su alimentación durante 3 días. Para el análisis de nutrientes, se seleccionaron los relacionados con los niveles de colesterol y la hipertensión y las cantidades mínimas diarias de nutrientes recomendadas en el Tercer Informe sobre el Monitoreo de la Nutrición en los Estados Unidos. Se usaron pruebas $t$ para comparar datos discretos (edad, escolaridad, ingesta de nutrientes e índice de masa corporal $\left(\mathrm{kg} / \mathrm{m}^{2}\right)$ y análisis de ji cuadrado $\left(\chi^{2}\right)$ para datos categóricos, como las percepciones de las encuestadas sobre su dieta y sus conocimientos. Además, los resultados se evaluaron mediante análisis de covarianza para ajustar las diferencias entre grupos por edad, ingreso como porcentaje de pobreza y escolaridad. Las diferencias se consideraron estadísticamente significativas al nivel de $P<0,01$ para compensar el uso de datos no ponderados y los efectos del gran tamaño muestral.

Los siguientes resultados fueron estadísticamente significativos: las afroamericanas tenían menos educación formal, eran más jovenes, vivían en hogares con ingresos en el nivel de pobreza o menores y tenían un mayor índice de masa corporal que las blancas. También fue estadísticamente significativo un mayor consumo de energía y grasas saturadas en las mujeres blancas que en las afroamericanas. Menos de una tercera parte de todas las mujeres cumplían con las recomendaciones de reducir su ingesta diaria de energía a menos de 30\% en grasas y a menos de $10 \%$ en grasas saturadas. Las afroamericanas consumían cantidades significativamente más altas de colesterol que las blancas, que eran más propensas a consumir menos de $300 \mathrm{mg}$ de colesterol al día y a seguir las recomendaciones respecto de la ingesta de potasio, calcio y magnesio. Los análisis de covarianza mostraron que cuando los resultados se ajustaban por edad, porcentaje de pobreza y escolaridad, la raza no se relacionaba significativamente con la ingesta de energía, pero persistían las otras diferencias. En cuanto a percepciones, las afroamericanas fueron significativamente más propensas a indicar que necesitaban disminuir la ingestión de grasa total, grasa saturada, colesterol y sal. Las mujeres blancas estaban más al tanto de la relación entre el colesterol y las grasas y por lo menos una dolencia, y de que la dieta puede originar problemas de salud. Las afroamericanas fueron 
significativamente menos capaces de reconocer los alimentos que contienen mucha grasa y colesterol y sabían menos sobre sus propiedades, la información en las etiquetas y las fuentes de grasas y colesterol que las blancas.

Mientras que las altas tasas de enfermedades crónicas en las poblaciones minoritarias se han atribuido en parte a niveles socioeconómicos y educativos más bajos que los de otros grupos, en el estudio hubo pocas diferencias que se pudieran explicar de esa forma. Sin embargo, las mujeres afroamericanas tienden a exceder del peso normal con mayor frecuencia que las blancas y la obesidad es uno de los factores de riesgo más importantes de hipertensión y un factor de riesgo de cardiopatía coronaria y diabetes tipo 2 en mujeres. Otros estudios indican que las mujeres afroamericanas subestiman su propio tamaño y se sienten atractivas y satisfechas con su apariencia aunque pesen más de lo normal. El estudio ha revelado información relevante para iniciar programas de educación nutricional especialmente dirigidos a la mujer afroamericana. (Gates $G$, McDonald M. Comparison of dietary risk factors for cardiovascular disease in African-American and white women. J Am Diet Assoc 1997;97:1394-1400.)

\section{¿Es el selenio una nueva arma contra el cáncer de próstata?}

El selenio es un nutriente esencial que se encuentra en pequeñas cantidades en los granos, el pescado y las carnes. Este elemento entra en la cadena alimentaria por conducto de las plantas, en las cuales su concentración varía de acuerdo con la del suelo. Por esta razón, el selenio que se ingiere con la dieta varía mucho de una población a otra. Hace casi 30 años se publicó un estudio que planteaba la posibilidad de que las tasas regionales de mortalidad por cáncer en los Estados Unidos de América tuvieran una correlación inversa con la exposición al selenio (Shamberger RJ, Frost DV. Possible protective effect of selenium against human cancer. Can Med Assoc J 1969;100:682). Durante los decenios subsecuentes se ha investigado la relación entre la concentración del selenio en la sangre y el riesgo de desarrollar distintos tipos de cáncer. Desde el punto de vista epidemiológico, los resultados han sido promisorios pero inconcretos. Se han observado correlaciones inversas del selenio con cáncer del esófago, colorrecto, estómago y pulmones, pero no con el cáncer de mama. También se ha observado en modelos animales que, a concentraciones relativamente altas, el selenio protege contra ciertos carcinógenos.
Hasta hace poco no se había establecido ninguna asociación con el cáncer de próstata, pero un nuevo ensayo aleatorio ha cambiado la situación. En un estudio de pacientes con antecedentes de carcinomas de células basales y escamosas, que vivían en zonas de los Estados Unidos con bajo contenido de selenio en el suelo, los hombres que recibieron $200 \mu \mathrm{g}$ de selenio al día por un promedio de 4,5 años tuvieron un riesgo de cáncer de próstata significativamente menor que los que tomaron placebo $(P=0,001)$. La reducción del riesgo se observó en presencia de cánceres en estadios A y B y más avanzados, así como en hombres con concentraciones iniciales de antígeno prostático específico menores y también mayores de $4 \mathrm{ng} / \mathrm{mL}$. La exclusión de casos diagnosticados en los primeros 2 años aumentó el efecto del tratamiento. La administración de selenio no protegió contra el desarrollo de cánceres de la piel como resultado primario, pero la incidencia de cáncer pulmonar y colorrectal tuvo reducciones notables. El estudio descrito se llevó a cabo en zonas de los Estados Unidos donde la ingesta diaria de selenio se estimó en $90 \mu \mathrm{g}$ al día (la recomendada oficialmente para los hombres es de $70 \mu \mathrm{g}$ y $75 \mu \mathrm{g}$ en el Reino Unido). Otro estudio en los Estados Unidos reveló una asociación inversa entre el cáncer de próstata avanzado y las concentraciones de selenio en las uñas de los pies, las cuales representan la ingesta de selenio a largo plazo. Las ingestas diarias de selenio estimadas sobre la base de su concentración en las uñas de los pies fueron de 86 a $159 \mu \mathrm{g}$.

Esos hallazgos apoyan fuertemente la suplementación de selenio para hombres que normalmente ingieren de 80 a $90 \mu \mathrm{g}$ al día. Los resultados se observarían con toda probabilidad en 3 años o menos. La cantidad suplementaria de selenio que se requiere no es tóxica y gran parte de la población ya la ingiere por vía de la dieta normal. En el Reino Unido la ingestión de selenio ha disminuido en los últimos decenios debido a la menor importación de harina proveniente de los Estados Unidos y el consumo de harina europea que contiene mucho menos selenio. En ese período, la incidencia de cáncer de próstata y la mortalidad ajustadas por edad han aumentado notablemente en Inglaterra y Gales, si bien es prematuro concluir que se deben a la disminución de selenio en la dieta. Puesto que el consumo de este elemento en la dieta normal no puede controlarse, quizá se requiera intervención gubernamental para mejorar su concentración en el organismo en grandes segmentos de población. En Finlandia, desde 1984 se ha añadido selenio a los fertilizantes por su escasez en los suelos. (Giovannucci E. Selenium and risk of prostate cancer [comentario]. Lancet 1998;352:755-756.) 